\title{
Multihop Relaying over IM/DD FSO Systems with Pointing Errors
}

\author{
Emna Zedini, Student Member, IEEE, and Mohamed-Slim Alouini, Fellow, IEEE
}

\begin{abstract}
In this paper, the end-to-end performance of a multihop free-space optical system with amplify-and-forward channelstate-information-assisted or fixed-gain relays using intensity modulation with direct detection technique over Gamma-Gamma turbulence fading with pointing error impairments is studied. More specifically, novel closed-form results for the probability density function and the cumulative distribution function of the end-to-end signal-to-noise ratio (SNR) are derived in terms of the Fox's $H$ function. Based on these formulas, closed-form bounds for the outage probability, the average bit-error rate (BER) of on-off keying modulation scheme, the moments, and the ergodic capacity are presented. Furthermore, using the momentsbased approach, tight asymptotic approximations at high and low average SNR regimes are derived for the ergodic capacity in terms of simple elementary functions. The obtained results indicate that the overall system performance degrades with an increase of the number of hops. The effects of the atmospheric turbulence conditions and the pointing error are also quantified. All the analytical results are verified via computer-based MonteCarlo simulations.
\end{abstract}

Index Terms-Free-space optical (FSO) communication, multihop relaying, atmospheric turbulence, pointing errors, average bit-error rate (BER), ergodic capacity.

\section{INTRODUCTION}

Free-space optical (FSO) communication has gained a significant research attention as a cost effective and wide bandwidth access technique operating at the unlicensed optical spectrum with high security level, relative to the traditional radio frequency (RF) transmission [1]-[3]. However, atmospheric pressure in the FSO links causes fluctuations in the refractive index, which is known as atmospheric turbulence, severely degrading the system performance particularly over distances of $1 \mathrm{~km}$ or longer [2]. Moreover, building sway introduces vibrations in the transmitted beam, leading to a misalignment between the transmitter and the receiver, known as pointing error that limits the performance of FSO links [1], [4].

Finding the proper model to characterize the FSO link is one of the prime concerns in FSO communication. Several statistical models such as Log-normal, and Gamma-Gamma have been introduced to describe the fading over FSO channels. The Log-normal distribution is considered to be only accurate under weak turbulence channel conditions [2]. On

E. Zedini and M.-S. Alouini are with the Computer, Electrical, and Mathematical Science and Engineering (CEMSE) Division, King Abdullah University of Science and Technology (KAUST) Thuwal, Makkah Province, Saudi Arabia (e-mails:\{emna.zedini, slim.alouini\}@kaust.edu.sa).

This work was supported in part by a grant from King Abdulaziz City of Sciences and Technology (KACST) number: AT-34-145.

Copyright (c) 2015 IEEE. Personal use of this material is permitted. However, permission to use this material for any other purposes must be obtained from the IEEE by sending a request to pubs-permissions@ieee.org. the other hand, the Gamma-Gamma PDF is a good model for atmospheric turbulence under both small and large scales atmospheric fluctuations and is presented in [5]. Different types of modulation schemes are employed in FSO communication systems such as pulse position modulation (L-PPM) and on-off keying (OOK). However, OOK is the dominant modulation scheme which is widely deployed in commercial FSO systems, mainly because of its simplicity and resilience to laser nonlinearity [4].

Multihop relaying, where several intermediate terminals relay the signal from the source terminal to the destination terminal [6], can be used over FSO links to mitigate turbulenceinduced fading and, hence, increasing the reliability of the FSO link. It is an efficient technique to expand the coverage of wireless networks with low power requirements and offer high data-rate at the end-to-end communication. [7] presents the performance analysis of a dual-hop mixed RF/FSO transmission system with pointing errors taken into account. In [8], the end-to-end capacity of dual-hop FSO communication systems employing amplify-and-forward (AF) relaying is studied. Selection combining (SC) and maximum ratio combining (MRC) diversity techniques for a dual-branch transmission system composed of a direct RF link and a dual-hop fixed gain relay RF/FSO system are investigated in [9]. In [6], the outage probability of a multihop FSO system with amplifyand-forward (AF) or decode-and-forward (DF) relays over strong turbulence fading channels is studied. Relay-assisted transmission over Log-normal turbulence-induced fading with path loss has been investigated in [10]. The performance of the FSO multihop system using CSI-assisted and fixed-gain relays over Gamma-Gamma turbulence under heterodyne detection technique with pointing errors has been analysed in [11]. In [12], the end-to-end performance of the multihop FSO system using channel-state-information (CSI)-assisted relays and fixed-gain relays over Gamma-Gamma turbulence under intensity modulation with direct detection (IM/DD) technique has been examined. However, the performance study carried out in [12] does not include the effect of the pointing error. Also, the ergodic capacity, being an important performance metric of primary concern in the design of FSO systems, is not studied.

In this paper, we extend the work presented in [12] to study the performance of a multihop FSO system employing CSI-assisted and fixed-gain relays over Gamma-Gamma turbulence-induced fading with pointing error impairments under IM/DD technique. Using the well-known inequality between harmonic and geometric means of positive random variables (RVs) [13], we derive novel closed-form results for the probability density function (PDF), and the cumulative 
distribution function (CDF) of the end-to-end signal-to-noise ratio (SNR) in terms of the Fox's $\mathrm{H}$ function. Based on these formulas, we derive closed-form bounds for the outage probability, the average bit error rate (BER) of OOK modulation scheme, the moments, and the ergodic capacity. Moreover, we present tight asymptotic approximations of the ergodic capacity at high and low average SNR regimes via a momentsbased approach. Computer simulations are also included to verify the accuracy of the presented mathematical analysis.

The remainder of the paper is organized as follows. In Section II, the PDF of the product of rational powers of statistically independent Gamma-Gamma distributions with pointing errors is derived in closed-form. The multihop FSO system operating over Gamma-Gamma fading channels with pointing errors under IM/DD technique is then introduced, and closed-form results to characterize the multihop FSO links including the PDF, the CDF, and the moments are presented in Section III. Subsequently, system performance metrics such as the outage probability, the average BER of OOK, and the ergodic capacity along with its asymptotic results are studied in Section IV. The obtained analytical expressions in the previous sections are numerically evaluated and interpreted in Section V. Finally, we review our main results and we draw some conclusions in Section VI.

\section{StatisticAl BACKGROUND}

In this section, we derive exact closed-form expression for the PDF of the product of rational powers of $N$ independent, but not necessarily identically distributed (i.n.i.d.), GammaGamma with pointing errors RVs.

Theorem 1 (PDF of the product of rational powers of Gamma-Gamma with pointing errors RVs): Let $Y \triangleq$ $\prod_{i=1}^{N} \gamma_{i}^{l_{i} / k}$, where $l_{1}, l_{2}, \ldots, l_{N}, k$ are positive integers, and $\gamma_{i}$ is a RV following the Gamma-Gamma model with pointing error impairments, with the PDF given by [1, Eq. (20)], [7, Eq. (1)]

$$
f_{\gamma_{i}}(\gamma)=\frac{\xi_{i}^{2}}{2 \Gamma\left(\alpha_{i}\right) \Gamma\left(\beta_{i}\right) \gamma} \mathrm{G}_{1,3}^{3,0}\left[\alpha_{i} \beta_{i} h_{i}\left(\frac{\gamma}{\mu_{i}}\right)^{\frac{1}{2}} \mid \begin{array}{c}
\xi_{i}^{2}+1 \\
\xi_{i}^{2}, \alpha_{i}, \beta_{i}
\end{array}\right],
$$

where $\xi_{i}$ denotes the ratio between the equivalent beam radius at the receiver and the pointing error displacement standard deviation (jitter) at the receiver [1], [14] given as $\xi_{i}=\frac{w_{z_{e q}, i}}{2 \sigma_{s, i}}$, with $\sigma_{s, i}^{2}$ is the jitter variance at the receiver and $w_{z_{e q}, i}$ is the equivalent beam radius at the receiver [15], [16] (for negligible pointing errors, $\xi \rightarrow \infty), h_{i}=\frac{\xi_{i}^{2}}{\xi^{2}+1}, G_{., .}(\cdot)$ is the Meijer's $\mathrm{G}$ function as defined in [17, Eq. (9.301)], $\mu_{i}$ stands for the electrical SNR and is related to the average SNR $\bar{\gamma}_{i}$ such that $\mu_{i}=\bar{\gamma}_{i} \alpha_{i} \beta_{i} \xi_{i}^{2}\left(\xi_{i}^{2}+2\right) /\left[\left(\alpha_{i}+1\right)\left(\beta_{i}+1\right)\left(\xi_{i}^{2}+1\right)^{2}\right]$, and $\alpha_{i}$ and $\beta_{i}$ are the fading/scintillation parameters related to the atmospheric turbulence conditions [4], [18]. More specifically, assuming a plane wave propagation with aperture averaging, these parameters can be determined from the Rytov variance as [19], [20]

$$
\begin{aligned}
\alpha_{i} & =\left[\exp \left(\frac{0.49 \sigma_{R, i}^{2}}{\left(1+0.18 d_{i}^{2}+0.56 \sigma_{R, i}^{12 / 5}\right)^{7 / 6}}\right)-1\right]^{-1} \\
\beta_{i} & =\left[\exp \left(\frac{0.51 \sigma_{R, i}^{2}\left(1+0.69 \sigma_{R, i}^{12 / 5}\right)^{-5 / 6}}{\left(1+0.9 d_{i}^{2}+0.62 d_{i}^{2} \sigma_{R, i}^{12 / 5}\right)^{5 / 6}}\right)-1\right]^{-1}
\end{aligned}
$$

where $\sigma_{R, i}^{2}=0.5 C_{n}^{2} k_{w}^{7 / 6} L_{i}^{11 / 6}$ denotes the Rytov variance, and $d_{i}^{2}=k_{w} D_{a}^{2} /\left(4 L_{i}\right)$, where $D_{a}$ is the diameter of the receiver aperture, $k_{w}=2 \pi / \lambda_{w}$ is the optical wave number, $\lambda_{w}$ is the wavelength, $L_{i}$ is the propagation distance, and $C_{n}^{2}$ refers to the index of refraction structure parameter varying from $10^{-17} \mathrm{~m}^{-2 / 3}$ for weak turbulence to $10^{-13} \mathrm{~m}^{-2 / 3}$ for strong turbulence. Then the PDF of the RV $Y$ can be derived in closed-form in terms of the Fox's $\mathrm{H}$ function as ${ }^{1}$

$$
\begin{aligned}
f_{Y}(y) & =\frac{k y^{-1} \prod_{i=1}^{N} \xi_{i}^{2}}{\prod_{i=1}^{N} \Gamma\left(\alpha_{i}\right) \Gamma\left(\beta_{i}\right)} \\
& \times \mathrm{H}_{N, 3 N}^{3 N, 0}\left[y^{k} \prod_{i=1}^{N}\left(\frac{\left(\alpha_{i} \beta_{i} h_{i}\right)^{2}}{\mu_{i}}\right)^{l_{i}} \mid \begin{array}{l}
\zeta_{1} \\
\zeta_{2}
\end{array}\right],
\end{aligned}
$$

with $\zeta_{1}=\left(\xi_{1}^{2}+1,2 l_{1}\right), \ldots,\left(\xi_{N}^{2}+1,2 l_{N}\right)$ and $\zeta_{2}=\left(\xi_{1}^{2}, 2 l_{1}\right)$, $\left(\alpha_{1}, 2 l_{1}\right),\left(\beta_{1}, 2 l_{1}\right), \ldots,\left(\xi_{N}^{2}, 2 l_{N}\right),\left(\alpha_{N}, 2 l_{N}\right),\left(\beta_{N}, 2 l_{N}\right)$.

Proof: See Appendix A.

It is worth to mention that an efficient MATHEMATICA ${ }^{\circledR}$ implementation for evaluating the Fox's $\mathrm{H}$ function is presented in [22].

\section{STATISTICAL CHARACTERISTICS OF THE END-TO-END SNR}

\section{A. System and Channel Models}

We consider an $N$-hop FSO wireless communication system which operates over independent and not identically distributed Gamma-Gamma fading channels with pointing error impairments under IM/DD with on-off keying (OOK). The source terminal $\mathrm{S}$ communicates with the destination terminal D through $N-1$ intermediate terminals $R_{1}, R_{2}, \ldots, R_{N-1}$ which relay the information signal only from one hop to the next, acting as non-regenerative relays. All relay terminals simultaneously receive and transmit in the same frequency band, and no latency is incurred in the whole chain of

${ }^{1}$ Note that, the PDF of $Y$ given in (4) can also be represented in terms of the Meijer's G function by means of using [21, Eqs. (2.4.5), (2.1.4) and (2.9.1)] as

$$
\begin{aligned}
f_{Y}(y) & =\frac{k y^{-1} \prod_{i=1}^{N} \xi_{i}^{2}\left(2 l_{i}\right)^{\alpha_{i}+\beta_{i}-2}}{(2 \pi)^{2 \sum_{i=1}^{N} l_{i}-N} \prod_{i=1}^{N} \Gamma\left(\alpha_{i}\right) \Gamma\left(\beta_{i}\right)} \\
& \times \mathrm{G}_{2 \sum_{i=1}^{6 \sum_{i=1}^{N} l_{i}, 0}, 6 \sum_{i=1}^{N} l_{i}}\left[y^{k} \prod_{i=1}^{N}\left(\frac{\left(\alpha_{i} \beta_{i} h_{i}\right)^{2}}{16 \mu_{i} l_{i}^{4}}\right)^{l_{i}} \mid \begin{array}{l}
\chi_{1} \\
\chi_{2}
\end{array}\right],
\end{aligned}
$$

where $\chi_{1}=\Delta\left(2 l_{N}, 1+\xi_{N}^{2}\right), \ldots, \Delta\left(2 l_{1}, 1+\xi_{1}^{2}\right)$ and $\chi_{2}=\Delta\left(2 l_{1}, \xi_{1}^{2}\right)$, $\Delta\left(2 l_{1}, \alpha_{1}\right), \Delta\left(2 l_{1}, \beta_{1}\right), \ldots, \Delta\left(2 l_{N}, \xi_{N}^{2}\right), \Delta\left(2 l_{N}, \alpha_{N}\right), \Delta\left(2 l_{N}, \beta_{N}\right)$. 
transmission. In this case, the received signal at the first intermediate relay, $R_{1}$, can be expressed as

$$
y_{1}=s_{1} x+n_{1}=\eta I_{1} x+n_{1},
$$

where $s_{1}=\eta I_{1}$ is the instantaneous intensity gain of the first hop, $\eta$ denotes the effective photo-current conversion ratio of the receiver, $I_{1}$ stands for the first hop irradiance, $x$ is the OOK signal with values 0 or 1 , and $n_{1}$ refers to the AWGN at the input of $R_{1}$ with zero mean and variance $N_{0,1}$. The signal $y_{1}$ is then multiplied by the gain $g_{1}$ of the first intermediate node $R_{1}$ and retransmitted to the second intermediate node $R_{2}$, where the received signal can be determined as

$$
y_{2}=g_{1} s_{2}\left(s_{1} x+n_{1}\right)+n_{2}=g_{1} \eta I_{2}\left(\eta I_{1} x+n_{1}\right)+n_{2},
$$

where $I_{2}$ is the irradiance at the second hop, and $n_{2}$ is the AWGN at the input of $R_{2}$. Then, the received signal at the destination terminal $\mathrm{D}$ can be written as

$$
y_{N}=\prod_{i=1}^{N} g_{i-1} s_{i} x+\sum_{i=1}^{N} n_{i} \prod_{k=i+1}^{N} g_{k-1} s_{k}, \quad g_{0}=1 .
$$

Therefore, the end-to-end SNR can be given as [23]

$$
\gamma_{\mathrm{end}}=\left[\prod_{i=1}^{N} g_{i-1}^{2} s_{i}^{2}\right] /\left[\sum_{i=1}^{N} N_{0, i}\left(\prod_{k=i+1}^{N} g_{k-1}^{2} s_{k}^{2}\right)\right] .
$$

\section{B. CSI-Assisted Relays}

One choice for the relay gain was proposed in [23] as $g_{i}^{2} \triangleq$ $1 / s_{i}^{2}$, where the relay just amplifies the incoming signal with the inverse of the channel intensity gain of the previous hop, regardless of the noise of that hop. As mentioned in [23], such a relay technique serves as a benchmark for all practical multihop systems employing non-regenerative relays. The endto-end SNR $\gamma_{\text {end }}$ can be thus derived from Eq. (8) as

$$
\gamma_{\text {end }} \triangleq\left(\sum_{i=1}^{N} \frac{1}{\gamma_{i}}\right)^{-1}
$$

where $\gamma_{i}=\eta^{2} I_{i}^{2} / N_{0, i}$ is the instantaneous SNR for the $i$ th hop following the Gamma-Gamma model with pointing error impairments, with the PDF given by (1). It is noteworthy to mention that the derived equivalent SNR in Eq. (9) is not easily tractable due to the difficulty in finding its statistics. However, an upper bound for the end-to-end SNR $\gamma_{\text {end }}$ can be derived by using the well-known inequality between harmonic and geometric means for $\gamma_{1}, \gamma_{2}, \ldots, \gamma_{N}$ given by [13]

$$
\mathcal{H}_{N} \leqslant \mathcal{G}_{N}
$$

where $\mathcal{H}_{N} \triangleq N\left(\sum_{i=1}^{N} 1 / \gamma_{i}\right)^{-1}$ and $\mathcal{G}_{N} \triangleq \prod_{i=1}^{N} \gamma_{i}^{1 / N}$ are the harmonic and geometric means, respectively. Therefore, an upper bound for the end-to-end SNR for an $N$-hop FSO system with CSI-assisted relays can be obtained as [13, Eq. (14)]

$$
\gamma_{\mathrm{end}} \leqslant \gamma_{\mathrm{ub}}=\frac{1}{N} \prod_{i=1}^{N} \gamma_{i}^{1 / N}
$$

The form of $\gamma_{\mathrm{ub}}$ is mathematically more tractable than that in (9) and can be efficiently used to study several end-to-end performance metrics of the multihop FSO system. Using (4) by setting $l_{i}=1$ and $k=N$ into it, the PDF of the end-to-end SNR $\gamma_{\mathrm{ub}}$ can be determined in closed-form as

$$
f_{\gamma_{\mathrm{ub}}}(\gamma)=\frac{N \gamma^{-1} \prod_{i=1}^{N} \xi_{i}^{2}}{\prod_{i=1}^{N} \Gamma\left(\alpha_{i}\right) \Gamma\left(\beta_{i}\right)} \mathrm{H}_{N, 3 N}^{3 N, 0}\left[\gamma^{N} N^{N} \prod_{i=1}^{N} \frac{\left(\alpha_{i} \beta_{i} h_{i}\right)^{2}}{\mu_{i}} \mid \begin{array}{c}
\kappa_{1} \\
\kappa_{2}
\end{array}\right]
$$

where $\kappa_{1}=\left(\xi_{1}^{2}+1,2\right), \ldots,\left(\xi_{N}^{2}+1,2\right)$ and $\kappa_{2}=\left(\xi_{1}^{2}, 2\right),\left(\alpha_{1}, 2\right),\left(\beta_{1}, 2\right), \ldots,\left(\xi_{N}^{2}, 2\right),\left(\alpha_{N}, 2\right),\left(\beta_{N}, 2\right)$. Then, utilizing [24, Eq. (2.25.2/2)], the CDF of $\gamma_{\mathrm{ub}}$ can be obtained as

$$
\begin{aligned}
F_{\gamma_{\mathrm{ub}}}(\gamma) & =\frac{N \prod_{i=1}^{N} \xi_{i}^{2}}{\prod_{i=1}^{N} \Gamma\left(\alpha_{i}\right) \Gamma\left(\beta_{i}\right)} \\
& \times \mathrm{H}_{N+1,3 N+1}^{3 N, 1}\left[\gamma^{N} N^{N} \prod_{i=1}^{N} \frac{\left(\alpha_{i} \beta_{i} h_{i}\right)^{2}}{\mu_{i}} \mid \begin{array}{l}
(1, N), \kappa_{1} \\
\kappa_{2},(0, N)
\end{array}\right]
\end{aligned}
$$

By exploiting the well-known inequality for positive RVs, an upper bound for the $n$th order moment of $\gamma_{\mathrm{ub}}$ can be expressed as

$$
\mathbb{E}\left[\gamma_{\mathrm{end}}^{n}\right] \leqslant \mathbb{E}\left[\gamma_{\mathrm{ub}}^{n}\right]
$$

where $\mathbb{E}[\cdot]$ denotes the expectation operator. Since the RVs $\gamma_{i}$ are independent, the above equation can be written as

$\mathbb{E}\left[\gamma_{\text {end }}^{n}\right] \leqslant \mathbb{E}\left[\gamma_{\text {ub }}^{n}\right]=N^{-n} \mathbb{E}\left[\prod_{i=1}^{N} \gamma_{i}^{n / N}\right]=N^{-n} \prod_{i=1}^{N} \mathbb{E}\left[\gamma_{i}^{n / N}\right]$.

For Gamma-Gamma fading channels with pointing error impairments, the moments $\mathbb{E}\left[\gamma_{i}^{n}\right]$ are specified as

$$
\mathbb{E}\left[\gamma_{i}^{n}\right]=\int_{0}^{\infty} \gamma_{i}^{n} f_{\gamma_{i}}\left(\gamma_{i}\right) d \gamma_{i} .
$$

Substituting (1) in (16), using the RV transformation $z=\sqrt{\gamma_{i}}$, and applying [17, Eq. (7.811.4)], the moments of $\gamma_{i}$ reduce to the following simple expression

$$
\mathbb{E}\left[\gamma_{i}^{n}\right]=\frac{\xi_{i}^{2} \Gamma\left(2 n+\alpha_{i}\right) \Gamma\left(2 n+\beta_{i}\right)}{\Gamma\left(\alpha_{i}\right) \Gamma\left(\beta_{i}\right)\left(2 n+\xi_{i}^{2}\right)}\left[\frac{\left(\alpha_{i} \beta_{i} h_{i}\right)^{2}}{\mu_{i}}\right]^{-n} .
$$

Using (15) and (17), the moments of $\gamma_{\mathrm{ub}}$ can be obtained in closed-form as

$\mathbb{E}\left[\gamma_{\mathrm{ub}}^{n}\right]=\frac{1}{N^{n}} \prod_{i=1}^{N} \frac{\xi_{i}^{2} \Gamma\left(\frac{2 n}{N}+\alpha_{i}\right) \Gamma\left(\frac{2 n}{N}+\beta_{i}\right)}{\Gamma\left(\alpha_{i}\right) \Gamma\left(\beta_{i}\right)\left(\frac{2 n}{N}+\xi_{i}^{2}\right)}\left[\frac{\left(\alpha_{i} \beta_{i} h_{i}\right)^{2}}{\mu_{i}}\right]^{-\frac{n}{N}}$

Note that, the expression in (18) is useful to derive tight asymptotic approximations of the ergodic capacity at the low and high average SNR regimes, as will be shown in the next section. 


\section{Fixed-Gain Relays}

The fixed-gain relays offer simplicity and ease of deployment at the expense of performance, comparing to the CSIassisted relays [23]. Non-regenerative relays introduce fixed gains to the received signal given by $g_{i}^{2}=1 /\left(C_{i} N_{0, i}\right)$, where $C_{i}$ is a positive constant $\left(C_{0}=1\right)$. Using the same approach as in [23], the overall SNR at the destination can then be written as $[25]$

$$
\gamma_{\mathrm{end}}^{\prime}=\left(\sum_{i=1}^{N} \prod_{j=1}^{i} \frac{C_{j-1}}{\gamma_{j}}\right)^{-1}
$$

Utilizing Eq. (10), an upper bound for the end-to-end SNR when fixed-gain relays are employed, can be obtained as

$$
\gamma_{\mathrm{end}}^{\prime} \leqslant \gamma_{\mathrm{ub}}^{\prime}=\frac{1}{N} \prod_{i=1}^{N} C_{i}^{-\frac{(N-i)}{N}} \gamma_{i}^{\frac{N+1-i}{N}}
$$

Substituting $l_{i}=N+1-i$ and $k=N$ into (4) and performing some algebraic manipulations, the PDF of $\gamma_{\mathrm{ub}}^{\prime}$ can be derived in closed form as

$$
\begin{aligned}
f_{\gamma_{\mathrm{ub}}^{\prime}}(\gamma) & =\frac{N \gamma^{-1} \prod_{i=1}^{N} \xi_{i}^{2}}{\prod_{i=1}^{N} \Gamma\left(\alpha_{i}\right) \Gamma\left(\beta_{i}\right)} \\
& \times \mathrm{H}_{N, 3 N}^{3 N, 0}\left[\gamma^{N} N^{N} \prod_{i=1}^{N} C_{i}^{N-i}\left[\frac{\left(\alpha_{i} \beta_{i} h_{i}\right)^{2}}{\mu_{i}}\right]^{N+1-i} \mid \begin{array}{l}
J_{1} \\
J_{2}
\end{array}\right],
\end{aligned}
$$

where $J_{1}=\left(\xi_{1}^{2}+1,2 N\right), \ldots,\left(\xi_{N}^{2}+1,2\right)$ and $J_{2}=$ $\left(\xi_{1}^{2}, 2 N\right),\left(\alpha_{1}, 2 N\right),\left(\beta_{1}, 2 N\right), \ldots,\left(\xi_{N}^{2}, 2\right),\left(\alpha_{N}, 2\right),\left(\beta_{N}, 2\right)$.

Therefore, the CDF of $\gamma_{\mathrm{ub}}^{\prime}$ may be obtained by applying [24, Eq. (2.25.2/2)] with some algebraic manipulations as

$$
\begin{aligned}
& F_{\gamma_{\mathrm{ub}}^{\prime}}(\gamma)=\frac{N \prod_{i=1}^{N} \xi_{i}^{2}}{\prod_{i=1}^{N} \Gamma\left(\alpha_{i}\right) \Gamma\left(\beta_{i}\right)} \\
& \times \mathrm{H}_{N+1,3 N+1}^{3 N, 1}\left[\gamma^{N} N^{N} \prod_{i=1}^{N} C_{i}^{N-i}\left[\frac{\left(\alpha_{i} \beta_{i} h_{i}\right)^{2}}{\mu_{i}}\right]^{N+1-i} \mid \begin{array}{l}
(1, N), J_{1} \\
J_{2},(0, N)
\end{array}\right]
\end{aligned}
$$

Following the same approach as in the CSI-assisted relays case along with (17), we get the moments of $\gamma_{\mathrm{ub}^{\prime}}$ in closed-form in terms of simple functions as

$$
\begin{aligned}
\mathbb{E}\left[\gamma_{\mathrm{ub}}^{\prime n}\right] & =\mathcal{R}_{N}^{n} \prod_{i=1}^{N} \frac{\xi_{i}^{2} \Gamma\left(\frac{2(N+1-i) n}{N}+\alpha_{i}\right) \Gamma\left(\frac{2(N+1-i) n}{N}+\beta_{i}\right)}{\Gamma\left(\alpha_{i}\right) \Gamma\left(\beta_{i}\right)\left(\frac{2(N+1-i) n}{N}+\xi_{i}^{2}\right)} \\
& \times\left[\frac{\left(\alpha_{i} \beta_{i} h_{i}\right)^{2}}{\mu_{i}}\right]^{-\frac{(N+1-i) n}{N}}
\end{aligned}
$$

where $\mathcal{R}_{N}=\frac{1}{N} \prod_{i=1}^{N} C_{i}^{-\frac{(N-i)}{N}}$.

\section{Performance Metrics}

Based on the derived expressions in the previous section, we introduce closed-form bounds for the outage probability, the average BER of OOK, and the ergodic capacity for both CSI-assisted and fixed-gain relays.

\section{A. Outage Probability}

The outage probability is defined as the probability that the output SNR falls below a predetermined protection ratio $\gamma_{t h}$. Using (11), (13), (20), and (22), lower bounds for the outage probability can be obtained in closed-form such as $P_{\text {out }} \geqslant F_{\gamma_{\text {ub }}}\left(\gamma_{\text {th }}\right)$ when CSI-assisted relays are used, and $P_{\text {out }}^{\prime} \geqslant$ $F_{\gamma_{\mathrm{ub}}^{\prime}}\left(\gamma_{\mathrm{th}}\right)$ when fixed-gain relays are employed, respectively.

\section{B. Average BER}

The average BER of IM/DD with OOK can be expressed as $P(e)=P(0) P(e \mid 0)+P(1) P(e \mid 1)$, where $P(0)$ and $P(1)$ refer to the probabilities of transmitting 0 and 1 bits, respectively and $P(e \mid 0), P(e \mid 1)$ denote the conditional error probabilities when the bits 0 and 1 are transmitted, respectively. Assuming that $P(0)=P(1)=\frac{1}{2}$ and $P(e \mid 0)=P(e \mid 1)$, the conditional irradiance $I$ error probability can be given by [4], [26]

$$
P(e \mid I)=P(e \mid 0, I)=P(e \mid 1, I)=Q\left(\frac{\eta I}{\sqrt{2 N_{0}}}\right),
$$

where $Q(\cdot)$ is the Gaussian $Q$ function defined as $Q(x)=$ $(1 / \sqrt{2 \pi}) \int_{x}^{\infty} \exp \left(-t^{2} / 2\right) d t$ and can be written in terms of the complementary error function such that $\operatorname{erfc}(x)=2 Q(\sqrt{2} x)$. By averaging (24) over the irradiance $I$, the average BER $P(e)$ can be obtained as

$$
P(e)=\int_{0}^{\infty} P(e \mid I) f_{I}(I) d I .
$$

Using the relation between $I$ and the instantaneous end-toend SNR $\gamma_{\text {end }}$, i.e. $\gamma_{\text {end }}=\eta^{2} I^{2} / N_{0}$, the average BER can be formulated in terms of the SNR as

$$
P(e)=\frac{1}{2} \int_{0}^{\infty} \operatorname{erfc}\left(\frac{\sqrt{\gamma}}{2}\right) f_{\gamma_{\text {end }}}(\gamma) d \gamma .
$$

1) CSI-Assisted Relays: Substituting (12) into (26), representing $\operatorname{erfc}(\cdot)$ through the Fox's $H$ function $\operatorname{erfc}(\sqrt{\gamma} / 2)=1 / \sqrt{\pi} \mathrm{H}_{1,2}^{2,0}\left[\frac{\gamma}{4} \mid \begin{array}{c}(1,1) \\ (0,1),\left(\frac{1}{2}, 1\right)\end{array}\right]$ Eqs. (8.4.14/2) and (8.3.2/21)], and integrating using [24, Eq. (2.22.1/1)], a lower bound for the average BER of CSI-assisted relays over Gamma-Gamma fading channels with pointing errors can be shown to be given in closed-form in terms of the Fox's $\mathrm{H}$ function as

$$
\begin{aligned}
& P_{\gamma_{\mathrm{ub}}}(e)=\frac{N \prod_{i=1}^{N} \xi_{i}^{2}}{2 \sqrt{\pi} \prod_{i=1}^{N} \Gamma\left(\alpha_{i}\right) \Gamma\left(\beta_{i}\right)} \\
& \times \mathrm{H}_{N+2,3 N+1}^{3 N, 2}\left[(4 N)^{N} \prod_{i=1}^{N} \frac{\left(\alpha_{i} \beta_{i} h_{i}\right)^{2}}{\mu_{i}} \mid \begin{array}{c}
(1, N),\left(\frac{1}{2}, N\right), \kappa_{1} \\
\kappa_{2},(0, N)
\end{array}\right] .
\end{aligned}
$$

2) Fixed-Gain Relays: For a multihop FSO system equipped with fixed-gain relays, a lower bound on the average BER can be found by substituting (21) in (26) and using [24, Eq. (2.25.1/1)], yielding

$$
\begin{aligned}
& P_{\gamma_{\mathrm{ub}}^{\prime}}(e)=\frac{N \prod_{i=1}^{N} \xi_{i}^{2}}{2 \sqrt{\pi} \prod_{i=1}^{N} \Gamma\left(\alpha_{i}\right) \Gamma\left(\beta_{i}\right)} \\
& \times \mathrm{H}_{N+2,3 N+1}^{3 N, 2}\left[(4 N)^{N} \prod_{i=1}^{N} C_{i}^{N-i}\left[\frac{\left(\alpha_{i} \beta_{i} h_{i}\right)^{2}}{\mu_{i}}\right]^{N+1-i} \mid \begin{array}{l}
a_{1}, J_{1} \\
J_{2}, a_{2}
\end{array}\right],
\end{aligned}
$$


where $a_{1}=(1, N),\left(\frac{1}{2}, N\right)$ and $a_{2}=(0, N)$.

\section{Ergodic capacity}

The ergodic capacity for FSO systems with IM/DD can be bounded by

$$
\bar{C} \triangleq \mathbb{E}\left[\ln \left(1+c \gamma_{\mathrm{end}}\right)\right],
$$

where $c$ is a constant equal to $c=e /(2 \pi)$ [27, Eq. (26)], [28, Eq. (7.43)]. Since $\gamma_{\mathrm{end}} \leq \gamma_{\mathrm{ub}}$ and $\gamma_{\mathrm{end}}^{\prime} \leq \gamma_{\mathrm{ub}}^{\prime}, \ln \left(1+\gamma_{\mathrm{end}}\right) \leq$ $\ln \left(1+\gamma_{\mathrm{ub}}\right)$ and $\ln \left(1+\gamma_{\mathrm{end}}^{\prime}\right) \leq \ln \left(1+\gamma_{\mathrm{ub}}^{\prime}\right)$, and therefore, upper bounds for the ergodic capacity of both CSI-assisted and fixed-gain relays can be derived.

1) CSI-Assisted Relays: Substituting (12) into (29), utilizing the Fox's $H$ function representation of $\ln (1+c \gamma)$ as $\mathrm{H}_{2,2}^{1,2}\left[c \gamma \mid \begin{array}{l}(1,1),(1,1) \\ (1,1),(0,1)\end{array}\right]$ [24, Eqs. (8.4.6/5) and (8.3.2/21)], then integrating using [24, Eq. (2.25.1/1)], the ergodic capacity of an $N$-hop FSO with IM/DD system employing CSI-assisted relays can be upper bounded as

$$
\begin{aligned}
& \bar{C}_{\gamma_{\mathrm{ub}}}=\frac{N \prod_{i=1}^{N} \xi_{i}^{2}}{\prod_{i=1}^{N} \Gamma\left(\alpha_{i}\right) \Gamma\left(\beta_{i}\right)} \\
& \times \mathrm{H}_{N+2,3 N+2}^{3 N+2,1}\left[\left(\frac{N}{c}\right)^{N} \prod_{i=1}^{N} \frac{\left(\alpha_{i} \beta_{i} h_{i}\right)^{2}}{\mu_{i}} \mid \begin{array}{l}
(0, N),(1, N), \kappa_{1} \\
\kappa_{2},(0, N),(0, N)
\end{array}\right] .
\end{aligned}
$$

An asymptotic approximation of the ergodic capacity in (30) at high average SNR can be obtained from the first derivative of the $n$th order moment of $\gamma_{\mathrm{ub}}$ [29, Eqs. (8) and (9)] as

$$
\bar{C}_{\gamma_{\mathrm{ub}}} \approx \log (c)+\left.\frac{\partial}{\partial n} \mathbb{E}\left[\gamma_{\mathrm{ub}}^{n}\right]\right|_{n=0} .
$$

By substituting Eq. (18) into Eq. (31) and after some algebraic manipulations, the ergodic capacity can be asymptotically approximated at high average SNR as

$$
\begin{aligned}
& \bar{C}_{\gamma_{\mathrm{ub}} \mu_{i} \gg 1} \underset{1}{\approx} \log (c)-\log (N)+\frac{1}{N} \log \left(\prod_{i=1}^{N} \mu_{i}\right) \\
&+\frac{2}{N} \sum_{i=1}^{N}\left[\psi\left(\alpha_{i}\right)+\psi\left(\beta_{i}\right)-\log \left(\alpha_{i} \beta_{i} h_{i}\right)-\frac{1}{\xi_{i}^{2}}\right],
\end{aligned}
$$

where $\psi(\cdot)$ is the psi (digamma) function [17, Eq. (8.360.1)]. At low average SNR, the ergodic capacity is found to be approximated by the first moment. Evaluating (18) at $n=1$, we get the asymptotic approximation of the ergodic capacity at low average SNR in terms of simple functions as

$$
\begin{aligned}
\bar{C}_{\gamma_{\mathrm{ub}}} \underset{\mu_{i} \ll 1}{\approx} c \mathbb{E}\left[\gamma_{\mathrm{ub}}\right] \\
\quad=\frac{c}{N} \prod_{i=1}^{N} \frac{\xi_{i}^{2} \Gamma\left(\frac{2}{N}+\alpha_{i}\right) \Gamma\left(\frac{2}{N}+\beta_{i}\right)}{\Gamma\left(\alpha_{i}\right) \Gamma\left(\beta_{i}\right)\left(\frac{2}{N}+\xi_{i}^{2}\right)}\left[\frac{\left(\alpha_{i} \beta_{i} h_{i}\right)^{2}}{\mu_{i}}\right]^{-\frac{1}{N}} .
\end{aligned}
$$

2) Fixed-Gain Relays: For the case of fixed-gain relays, an upper bound for the ergodic capacity can be found after performing some algebraic manipulations using (21) and [24, Eq. $(2.25 .1 / 1)]$ as

$$
\begin{aligned}
& \bar{C}_{\gamma_{\mathrm{ub}}^{\prime}}=\frac{N \prod_{i=1}^{N} \xi_{i}^{2}}{\prod_{i=1}^{N} \Gamma\left(\alpha_{i}\right) \Gamma\left(\beta_{i}\right)} \\
& \times \mathrm{H}_{N+2,3 N+2}^{3 N+2,1}\left[\left(\frac{N}{c}\right)^{N} \prod_{i=1}^{N} C_{i}^{N-i}\left[\frac{\left(\alpha_{i} \beta_{i} h_{i}\right)^{2}}{\mu_{i}}\right]^{N+1-i} \mid \begin{array}{l}
b_{1}, J_{1} \\
J_{2}, b_{2}
\end{array}\right],
\end{aligned}
$$

where $b_{1}=(0, N),(1, N)$ and $b_{2}=(0, N),(0, N)$. At high average SNR, after performing some algebraic manipulations using (23), we get an accurate simple closed-form approximation of the ergodic capacity as

$$
\begin{aligned}
\bar{C}_{\gamma_{\mathrm{ub} \mu_{i} \gg 1} \underset{\sim}{\approx}} \log \left(c \mathcal{R}_{N}\right)+\log \left(\prod_{i=1}^{N} \mu_{i}^{\frac{N+1-i}{N}}\right) \\
\quad+\frac{2}{N} \sum_{i=1}^{N}(N+1-i)\left[\psi\left(\alpha_{i}\right)+\psi\left(\beta_{i}\right)-\log \left(\alpha_{i} \beta_{i} h_{i}\right)-\frac{1}{\xi_{i}^{2}}\right] .
\end{aligned}
$$

Furthermore, the ergodic capacity of a multihop FSO system using fixed-gain relays can be approximated in the low SNR regime in closed-form in terms of simple elementary functions by

$$
\begin{aligned}
\bar{C}_{\gamma_{\mathrm{ub} \mu_{i}^{\prime}} \ll 1} \approx \mathcal{R}_{N} \prod_{i=1}^{N} \frac{\xi_{i}^{2} \Gamma\left(\frac{2(N+1-i)}{N}+\alpha_{i}\right) \Gamma\left(\frac{2(N+1-i)}{N}+\beta_{i}\right)}{\Gamma\left(\alpha_{i}\right) \Gamma\left(\beta_{i}\right)\left(\frac{2(N+1-i)}{N}+\xi_{i}^{2}\right)} \\
\times\left[\frac{\left(\alpha_{i} \beta_{i} h_{i}\right)^{2}}{\mu_{i}}\right]^{-\frac{(N+1-i)}{N}} .
\end{aligned}
$$

\section{Numerical Results and Discussion}

Assuming equal average SNRs per hop for all hops $\bar{\gamma}_{i}=$ $\bar{\gamma}$, the outage probability, the average BER, and the ergodic capacity of the multihop FSO system with CSI-assisted and fixed-gain relays using IM/DD technique under the effect of pointing errors are evaluated numerically and illustrated. Weak $(\alpha=2.902$ and $\beta=2.51)$, moderate $(\alpha=2.296$ and $\beta=$ $1.822)$, and strong ( $\alpha=2.064$ and $\beta=1.342)$ turbulent FSO channel conditions are considered in our study [20, Table I].

In Fig. 1, the end-to-end outage probability of a multihop FSO system using CSI-assisted relays is plotted as a function of the inverse normalized outage threshold, $\bar{\gamma} / \gamma_{\text {th }}$, under weak turbulent conditions with strong pointing error $\xi=1.1$. Monte-Carlo Simulations for both lower bounds and exact results, based on Eq. (11) and Eq. (9) respectively, of the outage probability are also presented. A perfect match between analytical and simulation results of the lower bounds can be seen from Fig. 1. It can also be observed that the outage performance degrades as the number of hops increases (i.e. the higher the values of $N$, the higher will be the outage probability). Moreover, it can be shown that the lower the values of $\bar{\gamma} / \gamma_{t h}$, the tighter the bounds are.

Fig. 2 depicts the end-to-end outage probability for the case of CSI-assisted relays as a function of $\bar{\gamma} / \gamma_{\text {th }}$ for different numbers of hops $N$ and for varying effects of the 


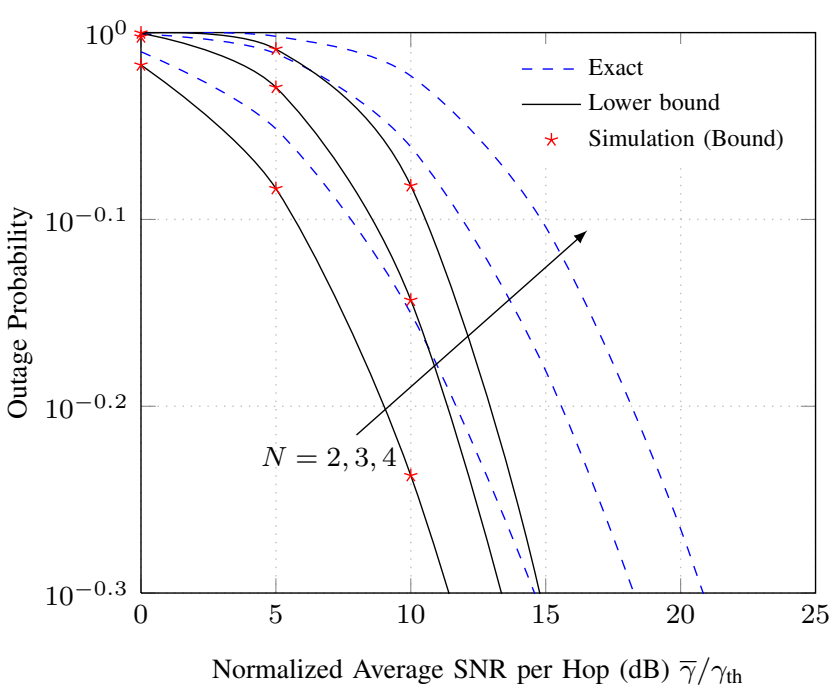

Fig. 1. Outage probability for a multihop FSO system using CSI-assisted relays with IM/DD under weak $(\alpha=2.902$ and $\beta=2.51)$ turbulent conditions with strong pointing error $(\xi=1.1)$.

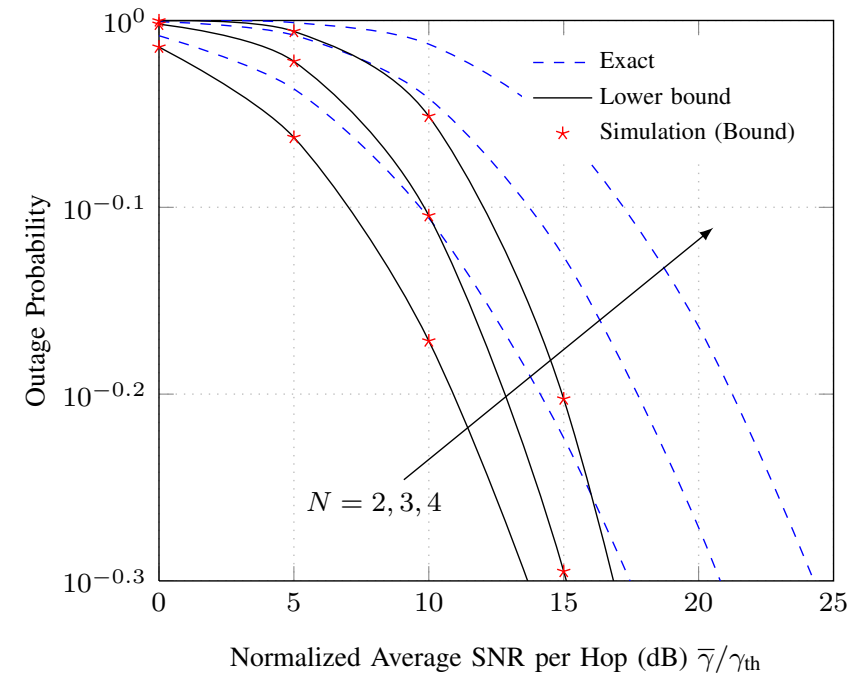

Fig. 2. Outage probability for a multihop FSO system using CSI-assisted relays with IM/DD for $C_{n}^{2}=5 \times 10^{-14} \mathrm{~m}^{-2 / 3}, L_{i}=3000+500(i-1)$, and $(\xi=2.1,1,6.7,1.1)$.

pointing error $(\xi=2.1,1,6.7,1.1)$. We consider that the refractive index is set such that $C_{n}^{2}=5 \times 10^{-14} \mathrm{~m}^{-2 / 3}$, the wavelength is equal $\lambda_{w}=1550 \mathrm{~nm}$, the aperture diameter for the receiver is $D_{a}=0.01 \mathrm{~m}$, and the distance between successive hops is $L_{i}=3000+500(i-1)(\mathrm{m})$, $i=1, \ldots, N$, resulting in different values of the fading parameters $((\alpha=(2.076,2.075,2.126,2.208)$ and $(\beta=$ $1.596,1.478,1.410,1.370)$ ). As clearly seen from the figure, our mathematical results are also verified when different $\alpha_{i}$, $\beta_{i}$, and $\xi_{i}$ are used for non-equidistant relays.

In Fig. 3, lower bounds on the end-to-end average BER of OOK are presented versus the average SNR per hop under strong, moderate, and weak turbulence conditions for $N=1$ and $N=3$. As clearly seen in the figure, the analytical and the simulation results for the lower bounds on the average BER are in a perfect agreement. We can also see from this figure that lower values of $N$ yield the best performance in terms of the average BER. Moreover, it can be observed that the average BER increases as the atmospheric turbulence conditions get severe.

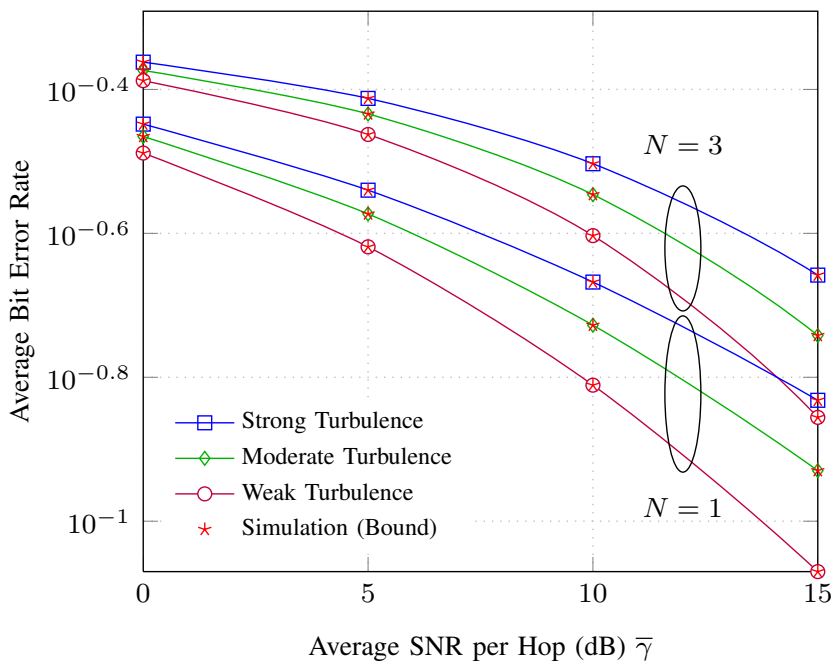

Fig. 3. Average BER of OOK for a multihop FSO system using CSI-assisted relays with IM/DD for $N=1$ and $N=3$ under weak $(\alpha=2.902$ and $\beta=2.51)$, moderate $(\alpha=2.296$ and $\beta=1.822)$, and strong $(\alpha=2.064$ and $\beta=1.342$ ) turbulence conditions for $\xi=6.7$.

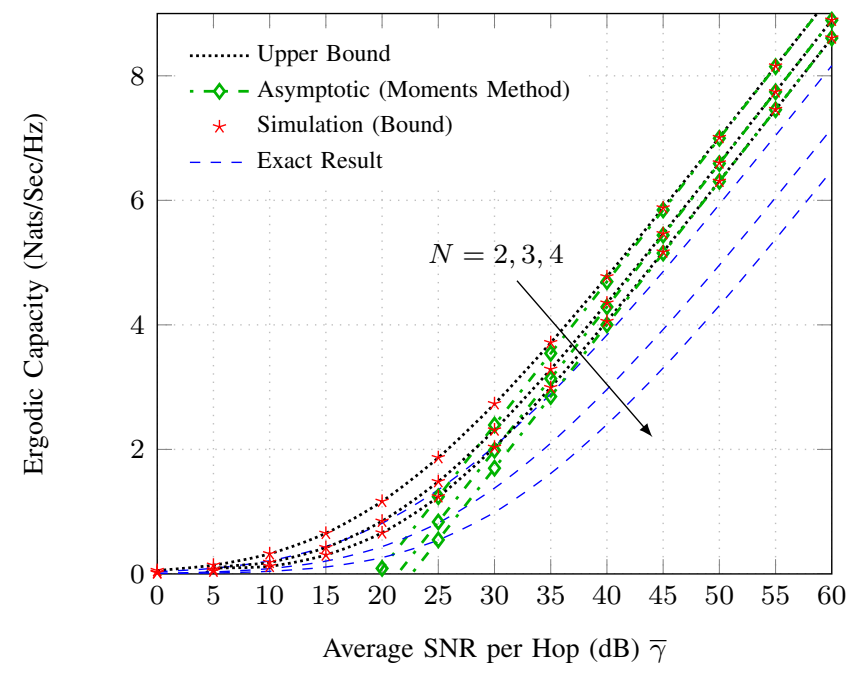

Fig. 4. Ergodic capacity for a multihop FSO system using CSI-assisted relays with IM/DD under strong turbulence $(\alpha=2.064$ and $\beta=1.342)$ with strong pointing error $(\xi=1.1)$.

Fig. 4 shows the ergodic capacity for strong turbulence conditions with strong pointing error $\xi=1.1$ using $N=2$, $N=3$, and $N=4$. As expected, increasing the number of hops decreases the ergodic capacity. Additionally, Fig. 4 indicates the tightness of the bound for lower values of $N$ even at high average SNR regime. Moreover, this figure shows the high accuracy of the asymptotic results based on the moments method derived in (32) at high average SNR values. 
As illustrated in Fig. 4, the analytical results for the bound on the ergodic capacity have also been verified by means of computer simulations.

The end-to-end outage probability of a multihop FSO system employing fixed-gain relays is plotted versus the inverse normalized outage threshold $\bar{\gamma} / \gamma_{\text {th }}$ in Fig. 5 for strong turbulence conditions under strong pointing error effects for several values of $N$. Monte-Carlo simulations for the exact results are also illustrated in the same figure showing the tightness and the accuracy of the bounds especially at low SNRs. Furthermore, it is observed that the outage probability degrades with an increase of the number of hops $N$ and shows a similar behaviour with the multihop FSO system with CSIassisted relays.

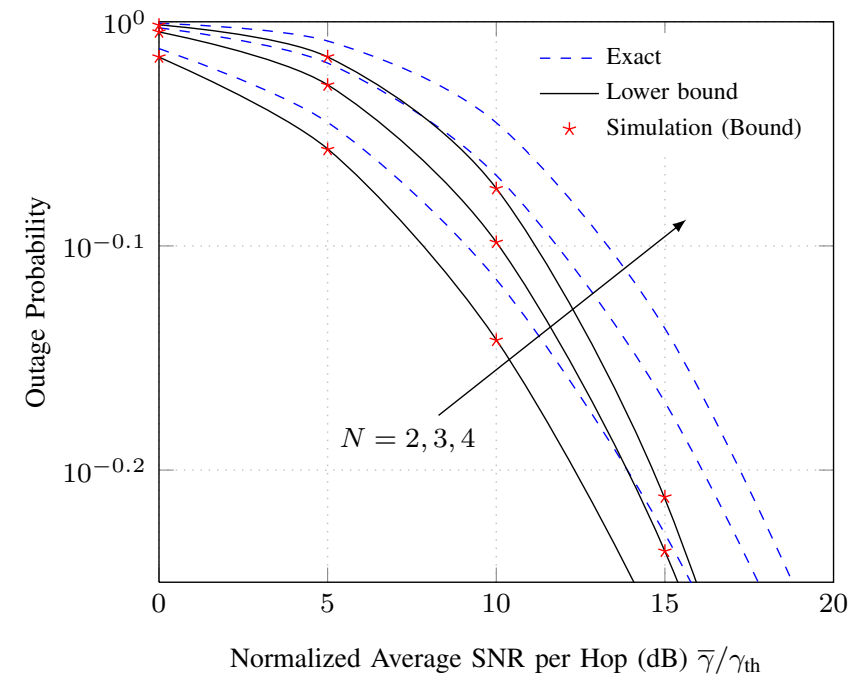

Fig. 5. Outage probability for a multihop FSO system using fixed-gain relays with IM/DD under strong $(\alpha=2.064$ and $\beta=1.342)$ turbulent conditions with strong pointing error $(\xi=1)$.

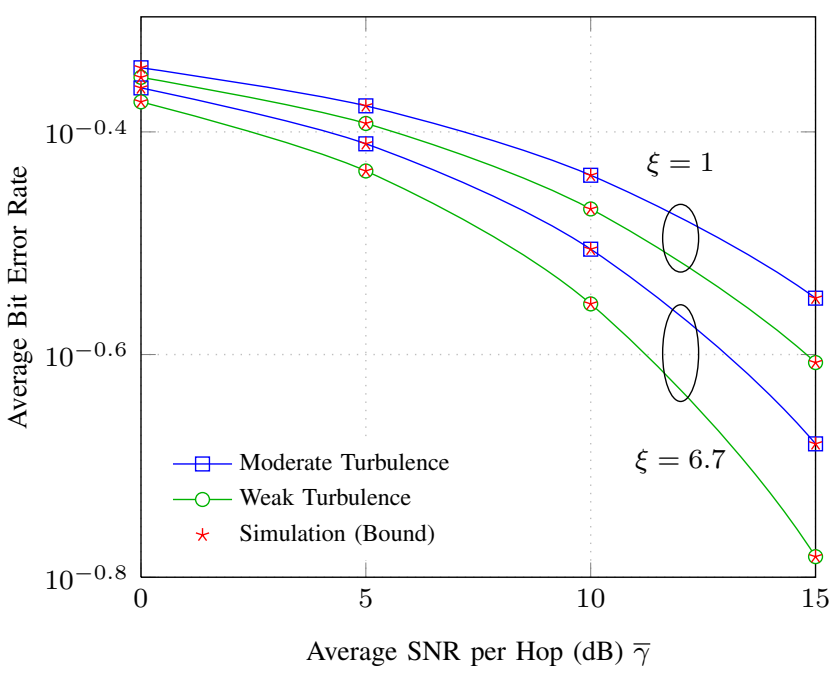

Fig. 6. Average BER of OOK for a multihop FSO system using fixed-gain relays with IM/DD for $N=4$ under weak $(\alpha=2.902$ and $\beta=2.51)$ and moderate $(\alpha=2.296$ and $\beta=1.822)$ turbulence conditions for varying effects of the pointing error.
In Fig. 6, the average BER of IM/DD with OOK for a multihop FSO system equipped with fixed-gain relays is illustrated for $N=4$. The obtained results indicate that the average BER performance deteriorates as the atmospheric turbulence conditions become severe. Equivalent results obtained via Monte-Carlo simulations are also included showing a perfect agreement with the analytical results. Moreover, as seen in this figure, when the effect of the pointing error decreases $(\xi \rightarrow \infty)$, then the average BER decreases leading to a system performance improvement, as expected.

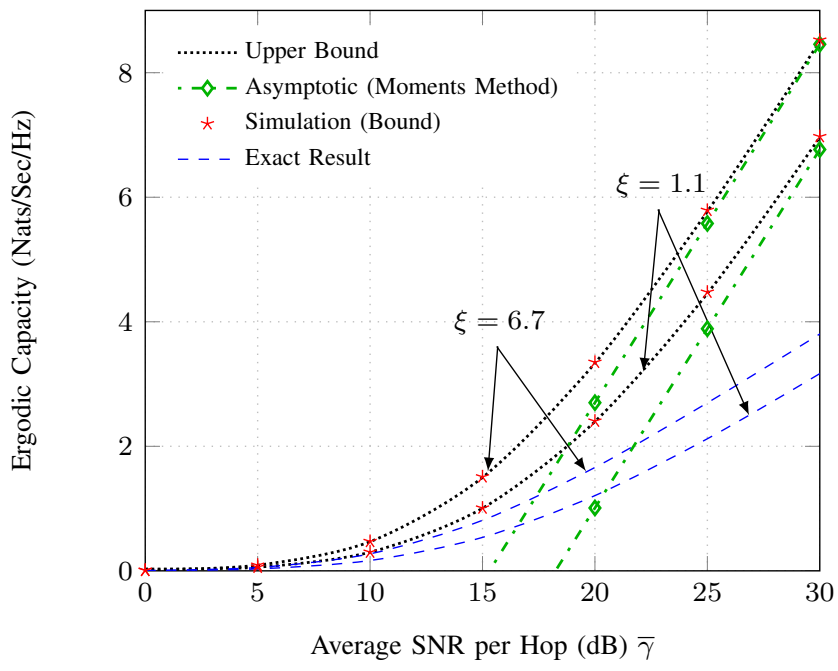

Fig. 7. Ergodic Capacity for a 4-hop FSO system using fixed-gain relays with IM/DD for strong turbulence $(\alpha, \beta)=(2.064,1.342)$.

Fig. 7 presents the end-to-end ergodic capacity of a 4-hop FSO system with fixed-gain relays under strong turbulence conditions for various pointing errors $(\xi=1.1$ and $\xi=6.7)$. As can be observed from this figure, the bounds are more tight for low values of $\bar{\gamma}$. However, the bounds lose tightness as $\bar{\gamma}$ increases. The accuracy of the asymptotic results at high average SNR ranges obtained via the moments-based approach by (35) is evident, especially at high average SNR values. Moreover, as it was expected, the ergodic capacity increases with the decrease of the pointing error effect.

\section{CONCLUSION}

In this paper, we have studied the performance of a multihop FSO system with IM/DD using AF CSI-assisted or fixed-gain relays over Gamma-Gamma fading channels under the effect of pointing errors. We have derived closed-form expressions for the PDF and the CDF of the end-to-end SNR in terms of the Fox's $\mathrm{H}$ function. Moreover, based on these formulas, the outage probability, the average BER of OOK modulation scheme, the moments, and the ergodic capacity were studied for different turbulent conditions and various values of the pointing error. Furthermore, new asymptotic approximations of the ergodic capacity at high and low average SNR were derived by applying the moments-based approach. Finally, our results demonstrate that the performance degrades as the pointing error effect and/or the atmospheric turbulent 
conditions become severe and with an increase of the number of hops.

\section{APPENDIX A \\ PROOF OF THEOREM 1}

In this appendix, we derive the PDF of $Y \triangleq$ $N^{-1} \prod_{i=1}^{N} \gamma_{i}^{l_{i} / k}$, where $\gamma_{i}$ is a RV whose PDF is given by (1). In order to obtain the PDF expression of $Y$, we first represent the PDF $f_{\gamma_{i}}(\gamma)$ of the Gamma-Gamma distribution with pointing error impairments in terms of the Fox's $\mathrm{H}$ function by means of some algebraic manipulations utilizing [21, Eq. (2.1.4)] and [21, Eq. (2.1.5)] together as

$$
\begin{aligned}
& f_{\gamma_{i}}(\gamma)=\frac{\xi_{i}^{2}\left(\alpha_{i} \beta_{i} h_{i}\right)^{2}}{\Gamma\left(\alpha_{i}\right) \Gamma\left(\beta_{i}\right) \mu_{i}} \\
& \times \mathrm{H}_{1,3}^{3,0}\left[\left.\frac{\left(\alpha_{i} \beta_{i} h_{i}\right)^{2} \gamma}{\mu_{i}}\right|_{\left(\xi_{i}^{2}-2,2\right),\left(\alpha_{i}-2,2\right),\left(\beta_{i}-2,2\right)}\right],
\end{aligned}
$$

where $\mathrm{H}_{., \cdot}(\cdot)$ is the Fox's $\mathrm{H}$ function [24, Eq. (8.3.1/1)]. Then, applying Theorem 4.2 from [30] and after performing some algebraic manipulations, the PDF of rational powers of Gamma-Gamma with pointing errors RVs, $Y_{1}=\gamma_{i}^{l_{i} / k}$, can be obtained in terms of the Fox's $\mathrm{H}$ function as

$$
\begin{aligned}
f_{Y_{1}}(y) & =\frac{\xi_{i}^{2}}{\Gamma\left(\alpha_{i}\right) \Gamma\left(\beta_{i}\right)}\left(\frac{\left(\alpha_{i} \beta_{i} h_{i}\right)^{2}}{\mu_{i}}\right)^{\frac{l_{i}}{k}} \\
& \times \mathrm{H}_{1,3}^{3,0}\left[\left(\frac{\left(\alpha_{i} \beta_{i} h_{i}\right)^{2}}{\mu_{i}}\right)^{\frac{l_{i}}{k}} y \mid \begin{array}{l}
\varrho_{1} \\
\varrho_{2}
\end{array}\right],
\end{aligned}
$$

where $\varrho_{1}=\left(\xi_{i}^{2}+1-\frac{2 l_{i}}{k}, \frac{2 l_{i}}{k}\right)$ and $\varrho_{2}=$ $\left(\xi_{i}^{2}-\frac{2 l_{i}}{k}, \frac{2 l_{i}}{k}\right),\left(\alpha_{i}-\frac{2 l_{i}}{k}, \frac{2 l_{i}}{k}\right),\left(\beta_{i}-\frac{2 l_{i}}{k}, \frac{2 l_{i}}{k}\right)$. Now, using Theorem 4.1 from [30] with some algebraic manipulations by means of employing [21, Eq. (2.1.4)] and [21, Eq. (2.1.5)] yields the desired PDF expression of $Y$ given in terms of the Fox's $\mathrm{H}$ function in (4).

\section{REFERENCES}

[1] W. Gappmair, "Further results on the capacity of free-space optical channels in turbulent atmosphere," IET Communications, vol. 5, no. 9 , pp. 1262-1267, Jun. 2011.

[2] L. C. Andrews, R. L. Phillips, and C. Y. Hopen, Laser Beam Scintillation with Applications. SPIE, 2001.

[3] W. Popoola and Z. Ghassemlooy, "BPSK subcarrier intensity modulated free-space optical communications in atmospheric turbulence," IEEE/OSA Journal of Lightwave Technology, vol. 27, no. 8, pp. 967973, Apr. 2009.

[4] H. Sandalidis, T. Tsiftsis, G. Karagiannidis, and M. Uysal, "BER performance of FSO links over strong atmospheric turbulence channels with pointing errors," IEEE Communications Letters, vol. 12, no. 1, pp. 44-46, Jan. 2008.

[5] A. Al-Habash, L. Andrews, and R. Phillips, "Mathematical model for the irradiance probability density function of a laser beam propagating through turbulent media," Optical Engineering, vol. 40, no. 8, pp. 1554 1562, Aug. 2001.

[6] T. Tsiftsis, H. Sandalidis, G. Karagiannidis, and N. Sagias, "Multihop free-space optical communications over strong turbulence channels," in Proceedings of IEEE International Conference on Communications (ICC, 2006), pp. 2755-2759.

[7] I. Ansari, F. Yilmaz, and M.-S. Alouini, "Impact of pointing errors on the performance of mixed RF/FSO dual-hop transmission systems," IEEE Wireless Communications Letters, vol. 2, no. 3, pp. 351-354, Jun. 2013.
[8] K. Peppas, A. Stassinakis, H. Nistazakis, and G. Tombras, "Capacity analysis of dual amplify-and-forward relayed free-space optical communication systems over turbulence channels with pointing errors," IEEE/OSA Journal of Optical Communications and Networking, vol. 5, no. 9, pp. 1032-1042, Sep. 2013.

[9] E. Zedini, I. S. Ansari, and M.-S. Alouini, "On the performance of hybrid line of sight RF and RF-FSO fixed gain dual-hop transmission systems," in IEEE Global Communications Conference (GLOBECOM' 14), Austin, TX, USA, Dec. 2014, pp. 2119-2124.

[10] M. Safari and M. Uysal, "Relay-assisted free-space optical communication," IEEE Transactions on Wireless Communications, vol. 7, no. 12, pp. 5441-5449, Dec. 2008.

[11] X. Tang, Z. Wang, Z. Xu, and Z. Ghassemlooy, "Multihop free-space optical communications over turbulence channels with pointing errors using heterodyne detection," Journal of Lightwave Technology, vol. 32, no. 15, pp. 2597-2604, Aug. 2014.

[12] C. Datsikas, K. Peppas, N. Sagias, and G. Tombras, "Serial free-space optical relaying communications over Gamma-Gamma atmospheric turbulence channels," IEEE/OSA Journal of Optical Communications and Networking, vol. 2, no. 8, pp. 576-586, Aug. 2010.

[13] G. Karagiannidis, T. Tsiftsis, and R. Mallik, "Bounds for multihop relayed communications in Nakagami-m fading," IEEE Transactions on Communications, vol. 54, no. 1, pp. 18-22, Jan. 2006.

[14] H. Sandalidis, T. Tsiftsis, and G. Karagiannidis, "Optical wireless communications with heterodyne detection over turbulence channels with pointing errors," Journal of Lightwave Technology, vol. 27, no. 20, pp. 4440-4445, Oct. 2009.

[15] A. Farid and S. Hranilovic, "Outage capacity optimization for free-space optical links with pointing errors," Journal of Lightwave Technology, vol. 25, no. 7, pp. 1702-1710, Jul. 2007.

[16] F. Yang, J. Cheng, and T. Tsiftsis, "Free-space optical communication with nonzero boresight pointing errors," IEEE Transactions on Communications, vol. 62, no. 2, pp. 713-725, Feb. 2014.

[17] I. S. Gradshteyn and I. M. Ryzhik, Table of Integrals, Series and Products. Academic, 2000.

[18] J. Park, E. Lee, and G. Yoon, "Average bit-error rate of the Alamouti scheme in Gamma-Gamma fading channels," IEEE Photonics Technology Letters, vol. 23, no. 4, pp. 269-271, 2011.

[19] L. C. Andrews and R. L. Phillips, Laser Beam Propagation Through Random Media. SPIE, 2005.

[20] X. Tang, Z. Ghassemlooy, S. Rajbhandari, W. Popoola, and C. Lee, "Coherent polarization shift keying modulated free space optical links over a Gamma-Gamma turbulence channel," American Journal of Engineering and Applied Sciences, vol. 4, no. 9, pp. 520-530, 2011.

[21] A. Kilbas and M. Saigo, H-Transforms: Theory and Applications. CRC, 2004.

[22] F. Yilmaz and M.-S. Alouini, "Product of the powers of generalized nakagami-m variates and performance of cascaded fading channels," in IEEE Global Telecommunications Conference (GLOBECOM'09), Nov. 2009, pp. $1-8$.

[23] M. Hasna and M.-S. Alouini, "Outage probability of multihop transmission over Nakagami fading channels," IEEE Communications Letters, vol. 7, no. 5, pp. 216-218, May. 2003.

[24] A. Prudnikov, Y. Brychkov, and O. Marichev, Integrals and Series, Volume 3: More Special Functions. CRC, 1999.

[25] G. Karagiannidis, "Performance bounds of multihop wireless communications with blind relays over generalized fading channels," IEEE Transactions on Wireless Communications, vol. 5, no. 3, pp. 498-503, Mar. 2006.

[26] S. Navidpour, M. Uysal, and M. Kavehrad, "BER performance of freespace optical transmission with spatial diversity," IEEE Transactions on Wireless Communications, vol. 6, no. 8, pp. 2813-2819, Aug. 2007.

[27] A. Lapidoth, S. Moser, and M. Wigger, "On the capacity of free-space optical intensity channels," IEEE Transactions on Information Theory, vol. 55, no. 10, pp. 4449-4461, Oct. 2009.

[28] S. Arnon, J. Barry, G. Karagiannidis, R. Schober, and M. Uysal, Advanced Optical Wireless Communication Systems. Cambridge University.

[29] F. Yilmaz and M.-S. Alouini, "Novel asymptotic results on the highorder statistics of the channel capacity over generalized fading channels," in Proceedings of IEEE International Workshop on Signal Processing Advances in Wireless Communications (SPAWC, 2012), 2012, pp. 389393.

[30] B. D. Carter and M. D. Springer, "The distribution of products, quotients and powers of independent h-function variates," SIAM Journal on Applied Mathematics, vol. 33, no. 4, pp. pp. 542-558, 1977. [Online]. Available: http://www.jstor.org/stable/2100749 